\title{
Small-scale turbulent fluctuations beyond Taylor's frozen-flow hypothesis
}

\author{
Xiaozhou He, ${ }^{1}$ Guowei He, ${ }^{2}$ and Penger Tong ${ }^{1, *}$ \\ ${ }^{1}$ Department of Physics, Hong Kong University of Science and Technology, Clear Water Bay, Kowloon, Hong Kong \\ ${ }^{2}$ LNM, Institute of Mechanics, Chinese Academy of Sciences, Beijing 100080, People's Republic of China
}

(Received 11 February 2010; revised manuscript received 30 April 2010; published 28 June 2010)

\begin{abstract}
The space-time cross-correlation function $C_{T}(r, \tau)$ of local temperature fluctuations in turbulent RayleighBénard convection is obtained from simultaneous two-point time series measurements. The obtained $C_{T}(r, \tau)$ is found to have the scaling form $C_{T}\left(r_{E}, 0\right)$ with $r_{E}=\left[(r-U \tau)^{2}+V^{2} \tau^{2}\right]^{1 / 2}$, where $U$ and $V$ are two characteristic velocities associated with the mean and rms velocities of the flow. The experiment verifies the theory and demonstrates its applications to a class of turbulent flows in which the requirement of Taylor's frozen flow hypothesis is not met.
\end{abstract}

DOI: 10.1103/PhysRevE.81.065303

PACS number(s): 47.27.eb, 05.40.-a, 44.25.+f

Fluid turbulence is often considered as a cascade process with turbulent kinetic energy being continuously transferred from large scales of system size $\ell_{0}$ to eddies of smaller size, until it dissipates when the eddy size becomes comparable to the viscous dissipation length $\ell_{d}$ [1]. An important quantity to describe this unique cascade process is the velocity crosscorrelation function

$$
C_{v}(r, \tau)=\langle v(x+r, t+\tau) v(x, t)\rangle_{t} /\left[\left(\sigma_{v}\right)_{1}\left(\sigma_{v}\right)_{2}\right],
$$

where $\left(\sigma_{v}\right)_{i}$ is the rms velocity at position $i$. For simplicity, here we only consider a one-dimensional case with separation $r$ being varied along the stream-wise $x$ direction. In theories of turbulence, the equal-time correlation function $C_{v}(r, 0)$ or its Fourier transform $E_{v}(k)$ is often used to describe the spectrum of turbulent kinetic energy in different lengths $r$ or wave numbers $k$. For example, according to Kolmogorov's 1941 theory [2], the kinetic energy is cascaded without loss through the inertial range, $\ell_{0}^{-1} \ll k \ll \ell_{d}^{-1}$, giving rise to a universal scale-invariant energy spectrum, $E_{v}(k) \sim \epsilon_{0}^{2 / 3} k^{-5 / 3}$, with $\epsilon_{0}$ being the constant energy dissipation rate.

Compared to the large number of theoretical studies, experimental information about small-scale turbulent fluctuations is often limited to the time series measurement of the local velocity at a single or a few spatial positions, from which one obtains the temporal correlation function $C_{v}(0, \tau)$ or its Fourier transform $E_{v}(f)$. While recent developments of particle image velocimetry allow one to obtain more spatial information for small-scale flows, time-domain measurements continue to play a major role in the experimental study of large-scale flows, such as turbulent jets and wind tunnels. To connect the time-domain results to the theoretical predictions made in the $k$-space, Taylor's frozen flow hypothesis [3] is often invoked in that turbulent fluctuations in space are assumed to be carried through a fixed location by a large convection flow $U_{0}$ without any significant change. Hence, one has $C_{v}(r, \tau)=C_{v}\left(r_{T}, 0\right)$ with

\footnotetext{
*penger@ust.hk
}

$$
r_{T}=r-U_{0} \tau
$$

Taylor's hypothesis requires a long correlation length and, thus, is applicable only to a special group of flows in which velocity fluctuations are much smaller than the mean flow velocity $U_{0}$. For many practical flows of interest, such as turbulent jets, channel flows and turbulent thermal convection where $U_{0}$ is comparable to or even becomes much smaller than $\sigma_{v}$, the requirement of the Taylor hypothesis is often not met $[4,5]$.

Recently, He et al. [6] proposed a model showing that $C_{v}(r, \tau)$ has a scaling form $C_{v}\left(r_{E}, 0\right)$ for a stationary and homogenous flow with $r_{E}$ being of the elliptical form

$$
r_{E}^{2}=(r-U \tau)^{2}+V^{2} \tau^{2} .
$$

Here $U$ is a characteristic convection velocity proportional to $U_{0}$ and $V$ is associated with a random sweeping velocity proportional to $\sigma_{v}$. Both $U$ and $V$ can be calculated from the second derivatives of $C_{v}(r, \tau)$ [6]. Equation (3) incorporates both the Taylor hypothesis when $V$ is small and Kraichnan's random sweeping hypothesis [7] for a homogenous and isotropic turbulent flow with a zero mean. He et al. derived Eq. (3) for small values of $r$ and $\tau$ and argued that it is also true for large values of $r$ and $\tau$ if the flow is scale invariant. They tested the model using the direct numerical simulation results obtained in a turbulent channel flow.

The scaling theory by $\mathrm{He}$ et al. has important practical implications for a large class of turbulent flows and thus it is essential to test the model in an actual flow system. In this Letter, we report direct measurements of the temperature cross-correlation function in turbulent Rayleigh-Bénard convection, where a fluid layer of thickness $H$ is heated from below and cooled from the top. For the Rayleigh number [8] $\mathrm{Ra} \gtrsim 10^{8}$, the convective flow becomes turbulent. The experiment confirms the theory and demonstrates it applications to turbulent flows beyond the Taylor frozen flow hypothesis.

The experiment is conducted in a upright cylindrical cell of inner diameter $D=19.0 \mathrm{~cm}$ and height $H=20.5 \mathrm{~cm}$ filled with water. Details about the apparatus and the experimental method have been described elsewhere [9], and here we mention only some key points. The sidewall of the cell is made of a transparent Plexiglas ring, which is sandwiched between the top and bottom brass plates. Two silicon rubber 
film heaters are used to provide uniform heating to the bottom plate. The top plate temperature is maintained constant by a temperature bath. The entire cell is placed inside a thermostat box, whose temperature matches the mean temperature of the bulk fluid, which is maintained at $40 \pm 0.3{ }^{\circ} \mathrm{C}$. The Prandtl number is then fixed at $\operatorname{Pr} \simeq 4.3$. The value of $\mathrm{Ra}$ is varied in the range of $10^{9} \lesssim \mathrm{Ra} \lesssim 10^{10}$, and here we focus on the measurements at $\mathrm{Ra}=1.4 \times 10^{10}$.

The flow in a closed convection cell is known to be inhomogeneous with a large-scale circulation (LSC) across the cell height [10]. In the rotation plane of the LSC, the flow is like a fly wheel with a zero mean velocity at the cell center and an increasing mean vertical velocity along the cell diameter at the midheight of the cell. After reaching its maximum value near the sidewall $(\sim 1 \mathrm{~cm}$ away from the cell wall), the mean vertical velocity starts to drop quickly and becomes zero at the cell wall. Therefore, the flow field near the sidewall is similar to that of a channel flow with a mean vertical velocity $U_{0}$ and a rms velocity $\sigma_{v} \simeq 0.6 U_{0}[10]$. For such a large rms velocity, Taylor's hypothesis is not expected to hold.

In the experiment, we use two small movable thermistors of $0.2 \mathrm{~mm}$ in diameter and $15 \mathrm{~ms}$ in time constant to measure temperature fluctuations at two locations. One is fixed at the middle height of the cell and is $2 \mathrm{~cm}$ away from the sidewall. The other thermistor is positioned above the fixed one with a varying vertical separation $+r$ along the down stream direction. The value of $r$ is varied from 0 to $16 \mathrm{~mm}$. For each value of $r$, we collect 10 -h-long time series data $\left(>10^{6}\right.$ data points) to ensure that the statistical averaging is adequate. The sampling rate of the temperature measurements is 40 Hz. From the simultaneous two-point measurements, we obtain the longitudinal temperature cross-correlation function

$$
C_{T}(r, \tau)=\langle\delta T(x+r, t+\tau) \delta T(x, t)\rangle_{t} /\left[\left(\sigma_{T}\right)_{1}\left(\sigma_{T}\right)_{2}\right],
$$

where $\delta T$ is the local temperature deviation from the mean and $\left(\sigma_{T}\right)_{i}$ is its standard deviation at position $i$.

It has been reported $[11,12]$ that temperature is an active scalar in turbulent convection only near the thermal boundary layers, where the temperature gradient is the largest. In the bulk region of the flow including the sidewall region, temperature is a passive scalar. In this case, the qualitative picture of energy cascade can also be used to describe the spectrum of temperature variance, because it is the turbulent motion that mixes the temperature field [13]. Therefore, the velocity and temperature fluctuations share the same decorrelation mechanism [14] and $C_{T}(r, \tau)$ is expected to have approximately the same scaling form as $C_{v}(r, \tau)$ does.

Figure 1(a) shows a three-dimensional (3D) plot of the measured $C_{T}(r, \tau)$ as a function of $r$ and $\tau$. The measured $C_{T}(r, \tau)$ is a single peaked function with a maximal value of $C_{T}(0,0)=1$ at the origin and decays to zero at large values of $r$ and $\tau$. Because of the limited range of $r$ varied, the measured $C_{T}(r, \tau)$ only decays to 0.65 at the largest $r$. Figure 1(b) shows a two-dimensional (2D) plot of isocorrelation contours of $C_{T}(r, \tau)$. In the range of $r$ and $\tau$ studied, the isocorrelation contours appear as a set of elliptical curves having the same orientation and aspect ratio. Such a common set of elliptical curves can be well described by Eq. (3) with con-
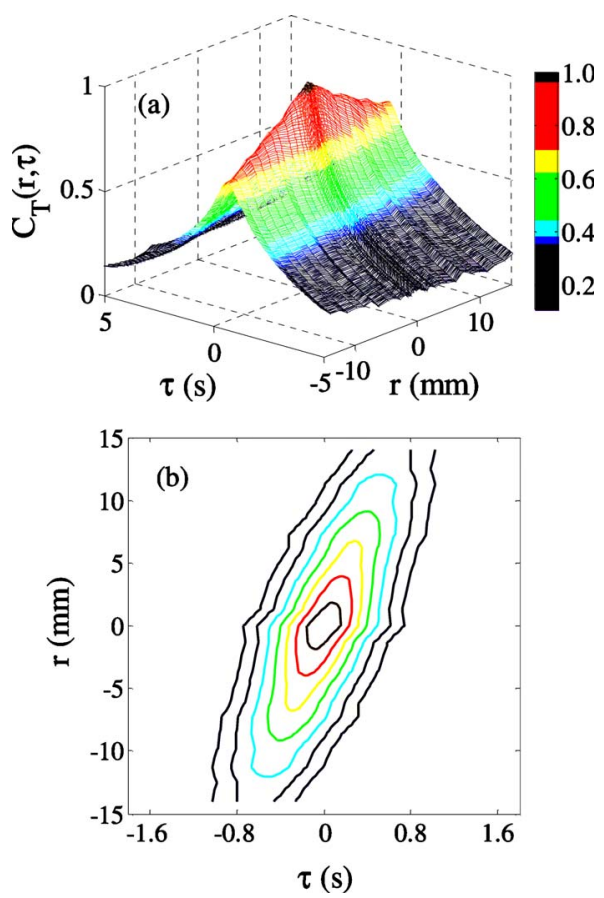

FIG. 1. (Color online) (a) 3D plot of the measured $C_{T}(r, \tau)$ as a function of $r$ and $\tau$. The correlation amplitude is color coded. (b) 2D plot of isocorrelation contours of $C_{T}(r, \tau)$ with the correlation amplitude varied from 0.95 to 0.65 at decrements of 0.05 (inner to outer contours). All the temperature measurements are made near the sidewall at $\mathrm{Ra}=1.4 \times 10^{10}$.

stant values of $r_{E}$. The orientation and aspect ratio of the elliptical circles are uniquely determined by the scaling velocities $U$ and $V$, which can be obtained directly from the measured $C_{T}(r, \tau)$.

It is seen from Fig. 1(a) that $C_{T}(r, \tau)$ as a function of $\tau$ at a fixed $r$ is a symmetric single peaked function with the peak position $\tau_{p}$ increasing with $r$. This is because it takes longer time for temperature fluctuations to move across a larger separation. Figure 2 shows the measured $\tau_{p}$ as a function of $r$, which is well described by a linear function, $\tau_{p}=\alpha r$, with $\alpha=4.36 \times 10^{-2} \mathrm{~s} / \mathrm{mm}$ (solid line). Because $C_{T}\left(r_{E}, 0\right)$ is a decreasing function of $r_{E}$, a minimum value of $r_{E}$ will give rise to a maximum value of $C_{T}\left(r_{E}, 0\right)$. Therefore, $\tau_{p}$ can be de-

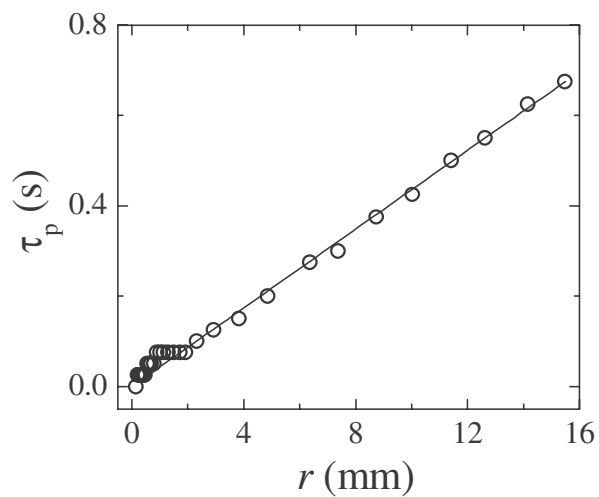

FIG. 2. Obtained peak position $\tau_{p}$ as a function of $r$. The solid line shows the fitted function, $\tau_{p}=\alpha r$, with $\alpha=4.36 \times 10^{-2} \mathrm{~s} / \mathrm{mm}$. 


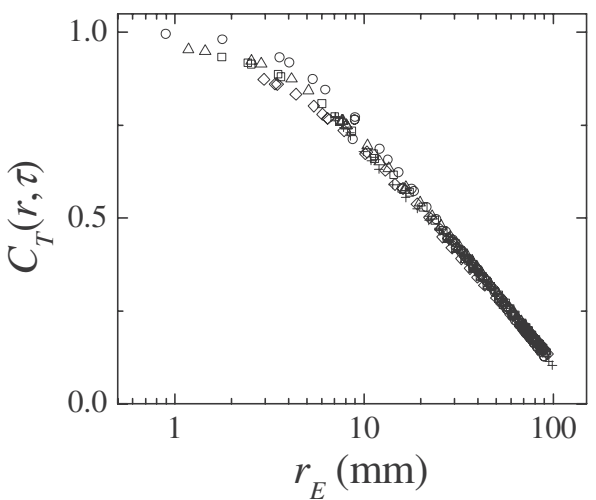

FIG. 3. Measured $C_{T}(r, \tau)$ as a function of the scaling variable $r_{E}$ for different values of $r: 0 \mathrm{~mm}$ (circles), $0.7 \mathrm{~mm}$ (triangles), 3.83 $\mathrm{mm}$ (squares), $8.76 \mathrm{~mm}$ (diamonds), and $15.49 \mathrm{~mm}$ (crosses). The value of $r_{E}$ is calculated using Eq. (3) with $U=14 \mathrm{~mm} / \mathrm{s}$ and $V=11.2 \mathrm{~mm} / \mathrm{s}$.

termined by the condition, $\partial r_{E} /\left.\partial \tau\right|_{r}=0$, from which we find $\tau_{p}=\left[U /\left(U^{2}+V^{2}\right)\right] r$. Similarly, we find the peak position $r_{p}$ from the $C_{T}(r, \tau) v s . r$ curve at a fixed $\tau$. The obtained $r_{p}$ is a linear function, $r_{p}=\beta \tau$, with $\beta=14 \mathrm{~mm} / \mathrm{s}$ (not shown). Using the condition, $\partial r_{E} /\left.\partial r\right|_{\tau}=0$, we find $r_{p}=U \tau$. Therefore, we have $U=14 \mathrm{~mm} / \mathrm{s}$ and $V=11.2 \mathrm{~mm} / \mathrm{s}$. The obtained value of $U$ is very close to the estimated mean velocity $U_{0} \simeq 15.6 \mathrm{~mm} / \mathrm{s}[10]$.

To further test the scaling form of $C_{T}(r, \tau)$, we plot, in Fig. 3, the measured $C_{T}(r, \tau)$ vs. $\tau$ curves at different values of $r$ as a function of $r_{E}$ using the obtained values of $U$ and $V$. All the correlation functions collapse into a single master curve, $C_{T}\left(r_{E}, 0\right)$, once the scaling variable $r_{E}$ is used. Figure 3 , thus, demonstrates that the measured $C_{T}(r, \tau)$ indeed has the predicted scaling form. When $V=0$, Eq. (3) becomes equivalent to the Taylor hypothesis, $r_{T}=r-U \tau$. It is found that the measured $C_{T}(r, \tau)$ vs $\tau$ curves at different values of $r$ do not scale with $r_{T}$, further confirming that the Taylor hypothesis does not hold in turbulent convection.

An important implication of Eq. (3) is that when $r=0$, we have $C_{T}(0, \tau)=C_{T}\left(r_{E}, 0\right)$ with

$$
r_{E}=\left(U^{2}+V^{2}\right)^{1 / 2} \tau \text {. }
$$

In this case, the single-point autocorrelation function $C_{T}(0, \tau)$ can be directly used to evaluate $C_{T}\left(r_{E}, 0\right)$ or its energy spectrum $E_{T}(k)$. Figures 4(a) and 4(b) show, respectively, the $C_{T}\left(r_{E}, 0\right)$ vs $r_{E} / \lambda_{T}$ and $E_{T}(k)$ vs $k \lambda_{T}$ curves obtained using the same time series data. Here, we have $k \lambda_{T}=\left(r_{E} / \lambda_{T}\right)^{-1}$ and the Taylor microscale $\lambda_{T}$ is used as the scaling length. Using the equation

$$
C_{T}\left(r_{E}, 0\right) \simeq 1-\left(r_{E} / \lambda_{T}\right)^{2}
$$

for small values of $r_{E}$, we obtain $\lambda_{T} \simeq 8.5 \mathrm{~mm}$ directly from the compensated plot of $C_{T}\left(r_{E}, 0\right)+\left(r_{E} / \lambda_{T}\right)^{2}$ vs $r_{E}$ (not shown). For the flow near the sidewall, one has [10] the shear rate $S \simeq 2 U_{0} / D \simeq 0.161 / \mathrm{s}$, the rms velocity $\sigma_{v}$ $\simeq 9.4 \mathrm{~mm} / \mathrm{s}$, and thus $[6] V \simeq\left[\left(S \lambda_{T}\right)^{2}+\sigma_{v}^{2}\right]^{1 / 2} \simeq 9.5 \mathrm{~mm} / \mathrm{s}$, which is close to the value of $V=11.2 \mathrm{~mm} / \mathrm{s}$ obtained above. In addition, we find the Reynolds number based on the Taylor microscale is $R e_{\lambda_{T}} \simeq\left(U^{2}+V^{2}\right)^{1 / 2} \lambda_{T} / \nu \simeq 240$.
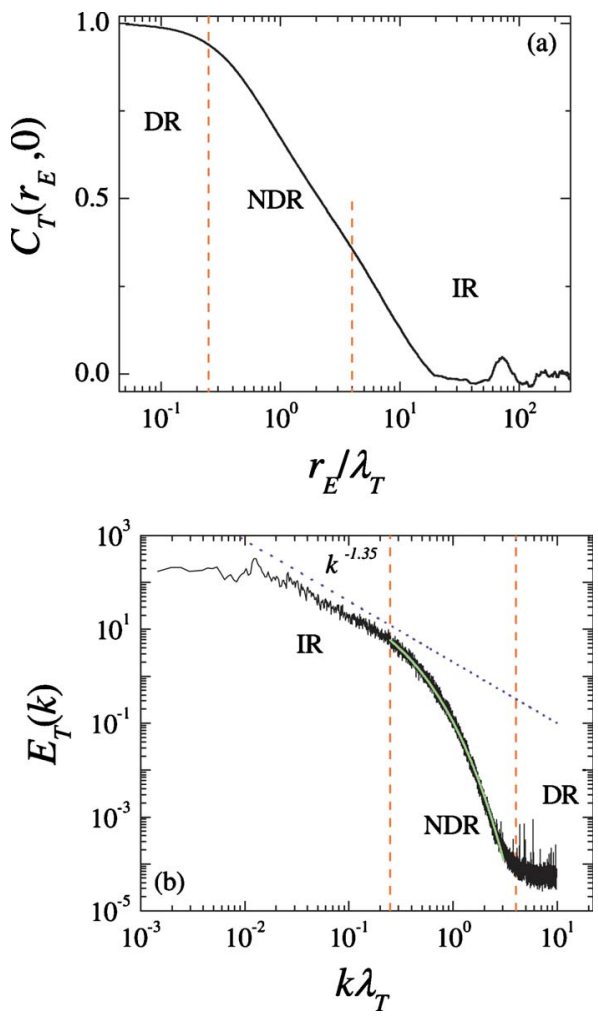

FIG. 4. (Color online) (a) Obtained $C_{T}\left(r_{E}, 0\right)$ as a function of $r_{E} / \lambda_{T}$. (b) Temperature power spectrum $E_{T}(k)$ as a function of $k \lambda_{T}$. The (blue) dotted line indicates the power law, $E\left({ }_{T}(k) \sim\left(k \lambda_{T}\right)^{-1.35}\right.$. The (green) dashed line is a plot of Eq. (8) with $a=129.4$ and $b=7.11$. The vertical (red) dashed lines indicate the three subranges of $r_{E} / \lambda_{T}$ or $k \lambda_{T}$ : the dissipation range (DR), near-dissipation range (NDR), and inertial range (IR).

Because of the high resolution of the temporal data, we are able to identify three distinct subranges of length scales from Fig. 4: the dissipation range (DR), near-dissipation range (NDR), and inertial range (IR). These three ranges are marked by the vertical dashed lines in Fig. 4 . The first two ranges, DR $\left(k \lambda_{T} \geqslant 4\right)$ and NDR $\left(0.25 \lesssim k \lambda_{T} \lesssim 4\right)$, are more clearly presented in the real space, whereas the last range, IR $\left(0.014 \lesssim k \lambda_{T} \lesssim 0.25\right)$, is more clearly viewed in the $k$ space. Such an identification of three distinct subranges in the same measurement clearly shows the power of the technique. While the single-point temperature power spectrum $E_{T}(f)$ [Fourier transform of $C_{T}(0, \tau)$ ] has been measured previously at the cell center and in the sidewall region [15-18], its connection to the energy spectrum $E_{T}(k)$ has not been firmly established in turbulent convection [18]. Previous attempts were made using Taylor's frozen flow hypothesis either explicitly or implicitly, but their validity was questioned in both the early and recent reviews $[18,19]$. With the scaling results shown in Fig. 3, Fig. 4 provides the first direct confirmation of $E_{T}(k)$ with the horizontal axis correctly labeled in units of $\lambda_{T}$.

In DR, $C_{T}\left(r_{E}, 0\right)$ has a parabolic form as shown in Eq. (6). In IR and NDR, all the measured $E_{T}(k)$ 's at different Ra superimpose with each other, once $k \lambda_{T}$ is used as the scaling variable. In IR, the measured $E_{T}(k)$ can be well described by a power law, 


$$
E_{T}(k) \sim\left(k \lambda_{T}\right)^{-\gamma},
$$

with the exponent $\gamma=1.35 \pm 0.05$ [(blue) dotted line in Fig. 4(b)]. A similar scaling result was also obtained in lowtemperature helium gas at $\mathrm{Ra} \simeq 10^{10}$ [16]. The obtained value of $\gamma$ is slightly smaller than the Corrsin-Oboukhov value of $\gamma=5 / 3$ for passive scalars in a turbulent flow at sufficiently large Reynolds numbers [13].

Figure 4(b) shows a small peak at $k \lambda_{T} \simeq 0.014$, below which $E_{T}(k)$ becomes featureless. This peak is caused by a weak temporal oscillation in the temperature field $[15,20]$. It was shown that the oscillation frequency corresponds to the inverse of the turnover time across the cell circumference [20]. This oscillation is convoluted into the measured $C_{T}\left(r_{E}, 0\right)$ in IR, making the measurement of the power law in real space difficult. In the $k$-space, however, the oscillation becomes a narrow peak, so that one can determine the exponent $\gamma$ more accurately.

NDR is located between DR and IR, in which $E_{T}(k)$ can be described by an stretched exponential function,

$$
E_{T}(k) \simeq a e^{-b\left(k \lambda_{T}\right)^{0.6}},
$$

with $a$ and $b$ as two fitting parameters. The (green) dashed line in Fig. 4(b) is a plot of Eq. (8) with $a=129.4$ and $b$ $=7.11$. The dynamics of temperature fluctuations in NDR are determined by a competition between the inertial and viscous forces [13]. Because of the limited range of scales available, it is difficult to uniquely determine the functional form of $E_{T}(k)$ in NDR. Other functional forms have also been proposed [21] and here we choose Eq. (8) for simplicity.

To conclude, we have shown that the measured $C_{T}(r, \tau)$ in the sidewall region has the scaling form $C_{T}\left(r_{E}, 0\right)$ as predicted in Eq. (3). We expect that this scaling behavior should be observable in a class of turbulent flows, which can be characterized by two scaling velocities associated with the mean and rms velocities of the flow. In this case, while the Taylor hypothesis is not valid, one can still use the scaling relation, $f t_{\lambda}=k \lambda_{T}$, to obtain a reliable power spectrum $E(k)$ from the single-point time-series data. Here, $f$ is frequency and $t_{\lambda}$ is Taylor's microtime, which can be determined from the measured $C_{T}(0, \tau) \simeq 1-\left(\tau / t_{\lambda}\right)^{2}$ at small $\tau$. We have also measured $C_{T}(r, \tau)$ at the cell center and found it does not have the predicted scaling form shown in Eq. (3). This may be due to the large-scale circulation (a fly-wheel-like flow), which introduces a linear shear in the region and thus makes the velocity field non-uniform in the central region. Extending the current theory to inhomogenous turbulent flows, such as turbulent shear flows, is an important task for future study.

The authors acknowledge support by the Hong Kong RGC under Grant No. HKUST-603208 (P.T.) and China NSFC under Project No. 10732090 (G.H.).
[1] U. Frisch, Turbulence: the Legacy of A. N. Kolmogorov (Cambridge University Press, Cambridge, UK, 1995).

[2] A. N. Kolmogorov, Dokl. Akad. Nauk SSSR 30, 9 (1941) [reprinted in Proc. R. Soc. London, Ser. A 434, 9 (1991)].

[3] G. I. Taylor, Proc. R. Soc. London, Ser. A 164, 476 (1938).

[4] J.-F. Pinton and R. Labbé, J. Phys. II 4, 1461 (1994).

[5] O. Chanal et al., Eur. Phys. J. B 17, 309 (2000).

[6] G.-W. He and J.-B. Zhang, Phys. Rev. E 73, 055303(R) (2006); X. Zhao and G.-W. He, ibid. 79, 046316 (2009).

[7] R. H. Kraichnan, Phys. Fluids 7, 1723 (1964).

[8] The Rayleigh number is defined as $\mathrm{Ra}=\alpha g \Delta T H^{3} /(\nu \kappa)$, where $g$ is the gravitational acceleration, $\Delta T$ is the temperature difference across the fluid layer of thickness $H$, and $\alpha, \nu$, and $\kappa$ are, respectively, the thermal expansion coefficient, the kinematic viscosity, and the thermal diffusivity of the fluid. The Prandtl number is defined as $\operatorname{Pr}=\nu / \kappa$.

[9] X.-Z. He and P. Tong, Phys. Rev. E 79, 026306 (2009).

[10] X.-L. Qiu and P. Tong, Phys. Rev. E 64, 036304 (2001).
[11] E. Calzavarini, F. Toschi, and R. Tripiccione, Phys. Rev. E 66, 016304 (2002)

[12] C. Sun, Q. Zhou, and K. Q. Xia, Phys. Rev. Lett. 97, 144504 (2006).

[13] H. Tennekes and J. L. Lumley, A First Course in Turbulence (MIT Press, Cambridge, MA, 1999).

[14] S.-Y. Chen and R. H. Kraichnan, Phys. Fluids A 1, 2019 (1989).

[15] B. Castaing et al., J. Fluid Mech. 204, 1 (1989).

[16] M. Sano, X. Z. Wu, and A. Libchaber, Phys. Rev. A 40, 6421 (1989).

[17] F. Chillá et al., EPL 22, 23 (1993).

[18] D. Lohse and K.-Q. Xia, Annu. Rev. Fluid Mech. 42, 335 (2010).

[19] E. Siggia, Annu. Rev. Fluid Mech. 26, 137 (1994).

[20] X.-L. Qiu and P. Tong, Phys. Rev. Lett. 87, 094501 (2001).

[21] T. Ishihara et al., J. Phys. Soc. Jpn. 74, 1464 (2005). 